\title{
Label-free nanoscopy enabled by coherent imaging with photonic waveguides
}

Florian Ströhl, Ida S. Opstad, Jean-Claude Tinguely, Firehun T. Dullo, Clemens F. Kaminski, et al.

Florian Ströhl, Ida S. Opstad, Jean-Claude Tinguely, Firehun T. Dullo, Clemens F. Kaminski, Balpreet S. Ahluwalia, "Label-free nanoscopy enabled by coherent imaging with photonic waveguides," Proc. SPIE 11076, Advances in Microscopic Imaging II, $110760 Z$ (22 July 2019); doi: 10.1117/12.2525702 


\title{
Label-free nanoscopy enabled by coherent imaging with photonic waveguides
}

\author{
Florian Ströhl ${ }^{1,2,}$, Ida S. Opstad ${ }^{1}$, Jean-Claude Tinguely ${ }^{1}$, Firehun T. Dullo ${ }^{1}$, Clemens F. \\ Kaminski $^{2}$, and Balpreet S. Ahluwalia ${ }^{1}$ \\ ${ }^{1}$ Department of Physics and Technology, UiT The Arctic University of Norway, NO-9037 \\ Tromsø, Norway \\ ${ }^{2}$ Department of Chemical Engineering and Biotechnology, University of Cambridge, CB3 0AS \\ Cambridge, UK
}

\begin{abstract}
In this project it was found that Fourier ptychographic microscopy can be improved far beyond its conventional limits via waveguide-based optical systems. Extensive in silico studies showed that images obtained on highrefractive index material waveguide chips in conjunction with hyperspectral illumination light and finely designed waveguide geometries can be combined via a modified phase-retrieval algorithm to yield a resolution below 150 $\mathrm{nm}$.
\end{abstract}

Keywords: label-free, nanoscopy, Fourier ptychography, waveguides, image reconstruction

\section{INTRODUCTION}

Label-free nanoscopy encompasses optical imaging with resolution in the 100nm range using visible wavelengths, vastly exceeding the Abbe limit of diffraction. A direct transfer of fluorescence-based nanoscopy to achieve this is not feasible due to a lack of access to non-linear sample responses. ${ }^{1}$ Hence, implementations have proven challenging. Here, we present a label-free nanoscopy concept based on purpose-made waveguide chips that replace conventional microscope slides. ${ }^{2}$ In combination with coherent imaging techniques ${ }^{3,4}$ the presented waveguidebased microscopy achieves a resolution approaching $150 \mathrm{~nm} .{ }^{5}$ Further optimization of the waveguide geometry and material is predicted to enable imaging close to the desired $100 \mathrm{~nm}$ mark. The developed photonic waveguide chips provide illumination at oblique angles, which "re-positions" the sample field's spectrum in the microscope aperture - mathematically, down-modulating the sample information with the illumination's frequency. Since this occurs before the objective's low-pass filtering, access to information outside the objective passband is gained. Due to the high spatial frequencies of the illumination provided by the chosen waveguide materials this can be far beyond the cut-off of conventional objectives. We validate the method via extensive simulations and provide details on the underlying image formation theory, the employed waveguide chips, as well as the reconstruction algorithm.

\section{IMAGING THEORY}

The commercial superresolution technique structured illumination microscopy ${ }^{6}$ achieves higher resolution by frequency mixing between the sample and illumination spatial frequencies. The degree of resolution enhancement is governed by the periodicity of the illumination stripe pattern. The larger the wave vector of the pattern, the higher the resulting resolution. A similar principle can be applied to scattering microscopy to overcome the coherent Abbe resolution limit ${ }^{3}$ via Fourier ptychography (FP). In conventional FP the length of the wave vector is limited by the wavelength and the numeric aperture, which together impose a resolution cap of about $200 \mathrm{~nm}$ to the technique. On photonic chips, however, the image resolution of FP can be enhanced much further than in its conventional implementation as the wave vector is artificially extended due to two effects. Firstly, the inherent geometry of the chip design allows for perfectly orthogonal evanescent wave illumination and secondly

*Correspondence: florian.strohl@uit.no 


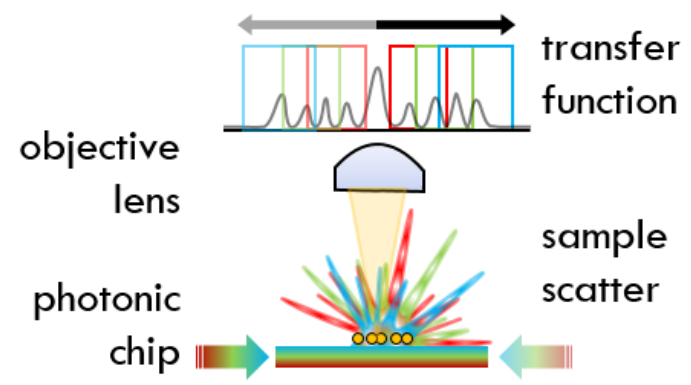

Figure 1. Artificially elongated illumination wavevectors grant access to higher spatial frequency information and can be combined via a dedicated phase retrieval algorithm.

the effective wavelength is also reduced by the waveguide material, which posses a high index of refraction. Example materials that can be used - silione nitride, tantalum-pentoxide, titanium-oxide, and even graphene or diamond - have refractive indices more than twice that of air, and can hence more than double the potential resolution enhancement. For instance, the use of $405 \mathrm{~nm}$ illumination light in conjunction with a photonic waveguide made from titanium-oxide $(\mathrm{n} \sim 2.5)$ and detection by a $0.95 \mathrm{NA}$ air objective results in an Abbe resolution of

$$
\frac{405 \mathrm{~nm}}{0.95+2.5}=117 \mathrm{~nm}
$$

Through multi-colour illumination impinging from a multitude of lateral directions, the extended sample spectrum can largely be covered by the raw image spectra, which can be linked to down-modulated parts of the actual sample spectrum (see Figure 1) and used for a Gerchberg-Saxton-style reconstruction. Details on the algorithm can be found elsewhere. ${ }^{7}$ It is important to note that enough overlap of the down-modulated spectra must be present for the algorithm to converge. To determine the effect of limited overlap due to design constraints, simulations have been performed that are presented below.

\section{SIMULATION RESULTS}

Simulations were performed assuming photonic chips made from silicon nitride and illuminated coherently with $488 \mathrm{~nm}, 561 \mathrm{~nm}$, and $647 \mathrm{~nm}$. The detection objective was set to be a $0.95 \mathrm{NA}$ air objective. Additionally to the evanescent illumination, coherent illumination was also simulated on-axis and obliquely as defined by $80 \%$ of the cut-off frequency of the detection objective. As shown in Figure 2, only the use of all three types of illumination permits an artefact-free reconstruction assuming a complex sample, whereas an amplitude-only sample might be reconstructed sufficiently well when only evanescent and on-axis illumination are used. Evanescent-only reconstructions were found to be a lot more prone to divergence of the algorithm, but could be processed differently. ${ }^{4}$

\section{DISCUSSION AND CONCLUSION}

In conclusion, we have presented on-chip Fourier ptychography and its use in label-free nanoscopy. Our results suggest that imaging with photonic waveguides does not permit artefact-free imaging on its own, but is reliant on additional illuminations, either coherently on-axis or with the further addition of oblique illumination directions. The former might be sufficient when imaging a sample with much greater response of the amplitude than of the phase, whereas the latter permits generally enhanced reconstruction results.

\section{ACKNOWLEDGMENTS}

\section{Funding information}

FS acknowledges funding from the European Molecular Biology Organisation (\#7411) and Marie SkłodowskaCurie actions (\#836355). BSA acknowledges funding from the European Research Council (\#336716). CFK 

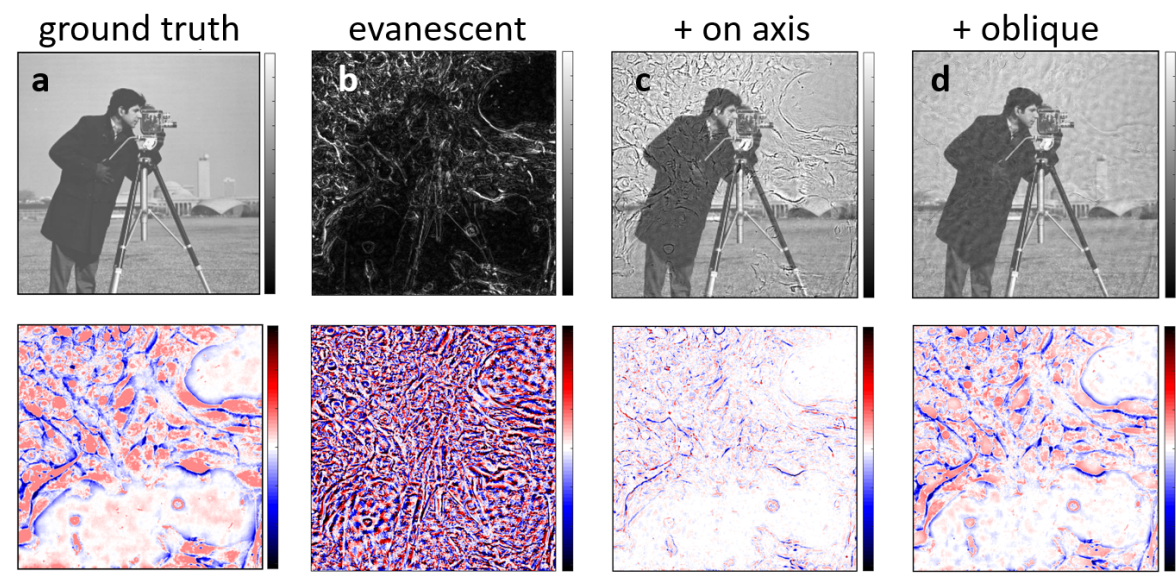

Figure 2. Simulation were performed on (a) a complex phantom with amplitude and phase components. Reconstruction from (b) only evanescent illuminations can not resolve any structures, while the addition of (c) an on-axis illuminated raw frame allows partial reconstruction of the amplitude part of the phantom. With the further addition of oblique illumination angles (d), also the phase can be retrieved successfully.

acknowledges funding from the Physical Sciences Research Council (EP/H018301/1), Medical Research Council (MR/K015850/1 and MR/K02292X/1), Wellcome Trust (089703/Z/09/Z), and Infinitus China Ltd.

\section{Competing interests statement}

BSA has applied for patent GB1705660.7 called Optical component for generating a periodic light pattern that covers the designs of the waveguide chip used in this work. The other authors declare no competing interests.

\section{REFERENCES}

[1] Schermelleh, L., Ferrand, A., Huser, T., Eggeling, C., Sauer, M., Biehlmaier, O., and Drummen, G. P., "Super-resolution microscopy demystified," Nature Cell Biology 21(1), 72 (2019).

[2] Diekmann, R., Helle, Ø. I., Øie, C. I., McCourt, P., Huser, T. R., Schüttpelz, M., and Ahluwalia, B. S., "Chip-based wide field-of-view nanoscopy," Nature Photonics 11(5), 322 (2017).

[3] Zheng, G., Horstmeyer, R., and Yang, C., "Wide-field, high-resolution fourier ptychographic microscopy," Nature photonics 7(9), 739 (2013).

[4] Ruh, D., Mutschler, J., Michelbach, M., and Rohrbach, A., "Superior contrast and resolution by image formation in rotating coherent scattering (rocs) microscopy," Optica 5(11), 1371-1381 (2018).

[5] Ströhl, F., Opstad, I. S., Tinguely, J.-C., Dullo, F. T., Mela, I., Osterrieth, J. W. M., Ahluwalia, B. S., and Kaminski, C. F., "A Super-Condenser for Labelfree Nanoscopy," arXiv e-prints , arXiv:1905.02401 (May 2019).

[6] Ströhl, F. and Kaminski, C. F., "Frontiers in structured illumination microscopy," Optica 3(6), 667-677 (2016).

[7] Pang, C., Li, J., Tang, M., Wang, J., Mela, I., Ströhl, F., Hecker, L., Shen, W., Liu, Q., Liu, X., Wang, Y., Zhang, H., Xu, M., Zhang, X., Liu, X., Yang, Q., and Kaminski, C. F., "On-chip super-resolution imaging with fluorescent polymer films," Advanced Functional Materials , 1900126 (2019). 